\title{
AN ALTERNATIVE SCHEME FOR STIFFENING SRF CAVITIES BY PLASMA SPRAYING
}

\author{
S.Bousson, M.Fouaidy, H.Gassot, T.Junquera, J.Lesrel, IPN Orsay, France \\ J.L Borne,J.Marini, LAL Orsay \\ C.Antoine, J.P.Charrier, H.Safa, DSM/DAPNIA/SEA CEA Saclay
}

\begin{abstract}
Stiffening of bulk niobium SRF cavities is mandatory for reducing the frequency shift induced by Lorentz forces at high accelerating gradients. Experimental and computational data previously reported show that with the actual scheme (i.e. EB welded stiffening rings) the frequency shift of TESLA 9 cells SRF cavities is higher than the cavity bandwidth above Eacc $=28 \mathrm{MV} / \mathrm{m}$. We propose a new stiffening method, using a Plasma Sprayed Copper Layer (PSCL) onto bulk niobium cavities. As compared to the actual technique, this method offers several advantages (simplicity, reliability...). The first experimental data obtained with monocell cavities produced by this method demonstrate the efficiency of cavities stiffening with plasma spraying. Thermal and mechanical properties measured on niobium samples with a PSCL are also presented. These data will allow us choose the plasma spraying process suitable for achieving the best cavities performances.
\end{abstract}

\section{INTRODUCTION}

Recent results obtained with 9-cells TESLA cavities point out a new problem for cavity stiffening. The actual EB welded stiffening rings are no more efficient for accelerating field above $28 \mathrm{MV} / \mathrm{m}$, Lorentz forces detuning becoming too important as compared to the cavity bandwidth. As cavities recently reached $33 \mathrm{MV} / \mathrm{m}$ [1], stiffening is already a problem and a solution has to be found. A new stiffening method is proposed, based on the coating of bulk $\mathrm{Nb}$ cavities by a plasma sprayed copper layer. The coating must be efficient for accelerating fields up to $40 \mathrm{MV} / \mathrm{m}$, which is the ultimate TESLA goal. Thanks to its good thermal conductivity, copper was the best material candidate to avoid cavity performances degradation. Mechanical characteristics of the copper coating could be close to bulk material with a suitable spraying process. As the Young modulus decreases strongly with the porosity, we have to find a spraying process which allows the lowest possible porosity (a few percent). Bond strength and achievable thickness are also very important issues. These properties are essential for choosing the more suitable spraying process.

\section{THERMAL SPRAYING TECHNIQUES}

The different thermal spraying methods can be divided into 3 different kinds [2].

\section{a) Plasma spraying.}

The principle is to create a plasma by an electric arc discharge initiated in a gas (usually $\mathrm{Ar} / \mathrm{H}_{2}$ ). The copper powder is injected in the high temperature plasma and the molten particles are sprayed out of the plasma gun. Depending on the spraying environment, different techniques were developed: under air (Atmospheric Plasma Spaying, APS), under inert gas (Controlled Atmosphere Plasma Spraying, CAPS), and under vacuum (Vacuum Plasma Spraying, VPS).

\section{b) Combustion flame spraying.}

The flame spraying (FS) principle is to use the chemical energy of combustion of fuel gas in oxygen to heat up the powder. If the oxygen is at a high pressure, the method is called High Velocity Oxy-Fuel Spraying (HVOF), and when an explosive mixture of oxygen and acetylene is used to post accelerate with the detonation (1-15 detonations per second), it is called Detonation-Gun Spraying (DGS).

c) Arc Spraying (AS).

Consumable electrodes made by two wires of the coating material are molten by arc heating, and the produced droplet is propelled by compressed gas. Some coating mechanical properties are summarised in Table 1.

Table 1: Main coating properties.

\begin{tabular}{|c|c|c|c|}
\hline Method & porosity & bond strength & Comments \\
\hline APS & medium & high & \\
\hline VPS & low & high & no oxidation \\
\hline CAPS & medium & high & no oxidation \\
\hline FS & high & low & \\
\hline DGS & low & high & pulsed \\
\hline HVOF & low & high & \\
\hline AS & high & low & \\
\hline
\end{tabular}

The first cavities were copper coated using the industrial APS method, and we are now working in a close collaboration with Ecole Nationale Superieure des Mines de Paris to improve this technique (lower porosity, higher bond strength without bonding layer). Moreover the HVOF and VPS methods are investigated with help of Institut Polytechnique de Sevenans (LERMPS, France). 


\section{RF TESTS ON Nb/Cu CAVITIES}

In order to study the feasibility of the new fabrication method, we performed RF tests on a $1.3 \mathrm{GHz}$ monocell cavity before and after the copper coating. Initially, the cavity was $2.5 \mathrm{~mm}$ thick, made from RRR $200 \mathrm{Nb}$ sheets, and then stiffened with a $2.5 \mathrm{~mm}$ thick copper layer. The coating was made by a "rough APS", a not optmised process, with the use of an intermediate $\approx 0.2 \mathrm{~mm}$ thick bonding layer (bronze/aluminium alloy) between niobium and copper. The two resulting Qo vs Eacc curves (Fig.1) show only a slight reduction of the maximum attainable field (quench), while the Qo level is almost not decreased.

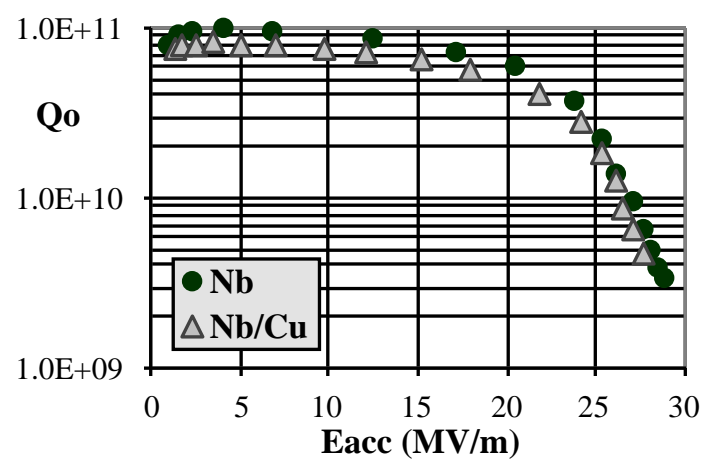

Fig. 1: Qo vs Eacc curves before and after copper coating on a $1.3 \mathrm{GHz}$ monocell cavity (C1 02) @ $1.7 \mathrm{~K}$.

During these experiments, the cavity frequency shift induced by Lorentz forces was measured. As theoretically expected, the frequency shift due to Lorentz forces depends quadratically on the accelerating field: $\Delta \mathrm{f}=\mathrm{K} . \mathrm{E}_{\text {acc }}{ }^{2}$, where $\mathrm{K}$ is a constant. On the Fig. 2, the $35 \%$ decrease of the slope of $\Delta f v_{\text {vs }}^{2}$ acc curve (Nb vs Nb with APS $\mathrm{Cu}$ coating) gives the stiffening efficiency of the APS copper coating.

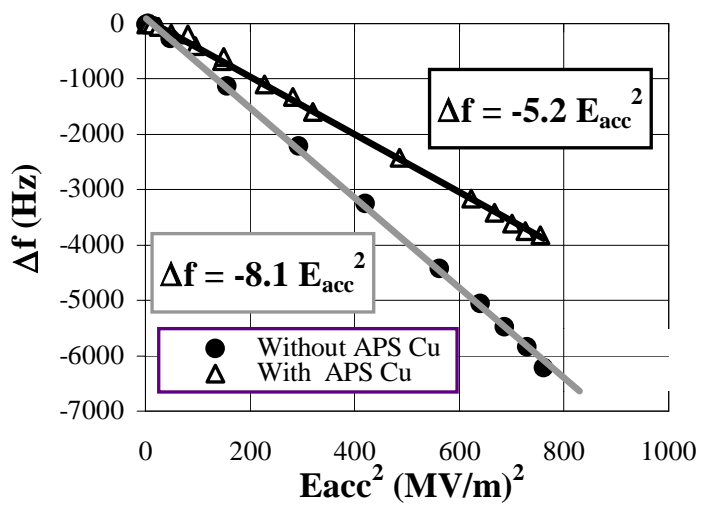

Fig $2: \Delta \mathrm{fvs}_{\text {acc }}{ }^{2}$ with and without APS Cu.

To carry on the study of the new stiffening method, five 3 $\mathrm{GHz}$ cavities were fabricated from RRR $40 \mathrm{Nb}$ sheets of $0.5 \mathrm{~mm}$ thickness [3]. The copper deposition process was first tested on the cavity \#3, and then two more cavities
(\#4 and \#5) were RF tested before and after $\mathrm{Cu}$ coating by the "rough APS" method. The results summarised in Table 2 show that the cavity performances are almost not modified by the APS Cu coating. Cavities \#1 and \#2 were tested before stiffening and reached high accelerating fields despite the poor $\mathrm{Nb}$ quality $(\mathrm{Eacc}=24.5 \mathrm{MV} / \mathrm{m}$ for cavity \#2). A study of several PS methods are currently in progress on samples in order to define the best stiffening method that will be tested on cavity \#1 and \#2.

Table 2: $3 \mathrm{GHz}$ cavity tests @ $1.8 \mathrm{~K}$.

\begin{tabular}{|c|c|c|}
\hline $\begin{array}{c}\text { Cavity } \\
\text { number }\end{array}$ & $\begin{array}{c}\mathrm{E}_{\text {acc }} \max \text { before } \mathrm{Cu} \\
\text { deposition }\end{array}$ & $\mathrm{E}_{\text {acc }}$ max with $\mathrm{Cu}$ \\
\hline$\# 1$ & $12.5 \mathrm{MV} / \mathrm{m}$ & to be tested \\
\hline$\# 2$ & $24.5 \mathrm{MV} / \mathrm{m}$ & to be tested \\
\hline$\# 3$ & not tested & $10 \mathrm{MV} / \mathrm{m}$ \\
\hline$\# 4$ & $16.5 \mathrm{MV} / \mathrm{m}$ & $16.5 \mathrm{MV} / \mathrm{m}$ \\
\hline$\# 5$ & $14.5 \mathrm{MV} / \mathrm{m}$ & $13.5 \mathrm{MV} / \mathrm{m}$ \\
\hline
\end{tabular}

\section{THERMAL INVESTIGATIONS}

The stiffening coating adds a supplementary thermal resistance $\Delta \mathrm{Rg}$ on the overall thermal resistance $(\mathrm{Rg})$ $\mathrm{Rg}=\mathrm{Rc}+\mathrm{Rk}+\Delta \mathrm{Rg}$, with $\mathrm{Rc}$ the conductivity term for the niobium and $\mathrm{Rk}$ the Kapitza resistance term (Kapitza resistance is nearly the same for $\mathrm{Nb}$ and $\mathrm{Cu}$ ). Thermal simulations were performed to determine the $\Delta \mathrm{Rg}$ threshold above which the cavity thermal behaviour could be modified (either by a maximum accelerating field decrease or by an effect on the Qo level). Both defect free case and defect case were studied. On the figure 4 is plotted the defect free case theoretical Qo vs Eacc for bulk $\mathrm{Nb}(\Delta \mathrm{Rg}=0)$. We have then calculated the Qo vs Eacc curves for an increase $\Delta \mathrm{Rg}$ of the overall thermal resistance, which is arbitrarily taken as equal to Rk and 3 $\mathrm{Rk}$. These two runs simulates a possible increase of $\mathrm{Rg}$ due to the copper coating.

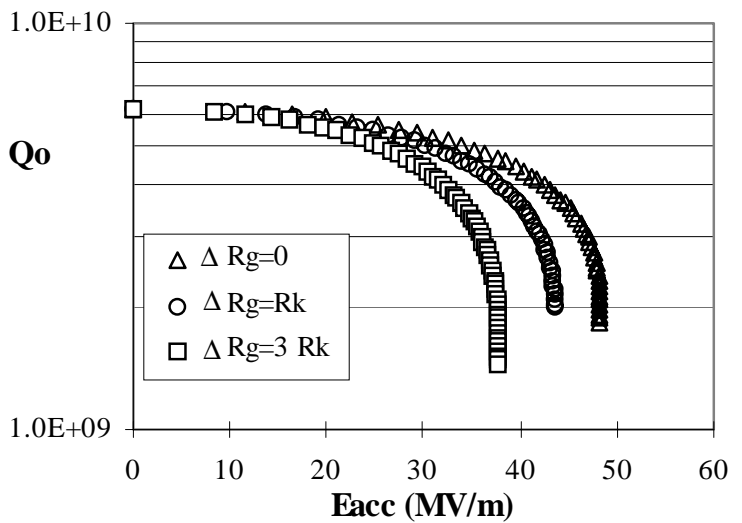

Fig. 4 : Simulation of Qo vs Eacc (defect free case) at $\mathrm{T}=2 \mathrm{~K} @ 1.3 \mathrm{GHz}$ with RRR=200 and 2.5mm thick.

The results clearly show that if $\Delta \mathrm{Rg}=\mathrm{Rk} \quad\left(=1.4 \quad 10^{-4}\right.$ W.m² $/ \mathrm{K} @ 2 \mathrm{~K}$ ), the TESLA goal $($ Eacc $=40 \mathrm{MV} / \mathrm{m})$ 
could be achieved, whereas if $\Delta \mathrm{Rg}=3 \mathrm{Rk}$, this goal is not reached. The defect case study leads to the same conclusion, so a threshold on the coating thermal resistance was determined for achieving the stiffening without drawbacks at $40 \mathrm{MV} / \mathrm{m}$.

In order to measure $\Delta \mathrm{Rg}$ due to the copper layer on our cavities, $\mathrm{Nb}$ samples were coated by the same "rough APS" process. $\triangle \mathrm{Rg}$ is obtained from the overall thermal resistance difference between a $\mathrm{Nb}$ sample and a $\mathrm{Cu}$ coated $\mathrm{Nb}$ sample [3]. At $1.8 \mathrm{~K}, \Delta \mathrm{Rg}$ was found to be $6.410^{-4} \mathrm{Km}^{2} / \mathrm{W}$ (about 4 times Rk). In this result, the effect of the bonding layer $(0.2 \mathrm{~mm}$ thick bronze/aluminium alloy) was suspected to dominate the thermal resistance, so another $\mathrm{Nb}$ sample coated with only the alloy coating was also measured. The result was the same $\left(\Delta \mathrm{Rg}=6.210^{-4} \mathrm{Km}^{2} / \mathrm{W}\right)$ clearly indicating the very low thermal conductivity of the bonding layer.

\section{MECHANICAL SIMULATIONS}

Numerical simulations have been performed to study the effect of different stiffening schemes using an additional copper layer on a TESLA 9-cells cavity detuning. The basics of the model are to consider a bimetal $\mathrm{Nb} / \mathrm{Cu}$ cavity (see Fig. 5), each material considered as bulk material perfectly bonded to each other [3].

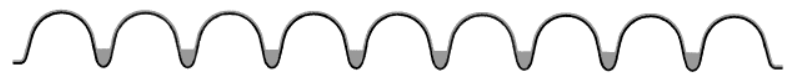

Fig. 5 : Stiffened bimetal $\mathrm{Nb} / \mathrm{Cu}$ cavity.

This ideal case was used to model different stiffening options, with homogeneous or non-homogeneous (thicker coating at the iris) copper layer, and Lorentz forces detuning @ Eacc=40 MV/m was computed for TESLA 9-cells cavities. The results are reported in Table 3. They show that with the actual stiffening scheme $(2.5 \mathrm{~mm} \mathrm{Nb}$ thickness and EB welded stiffening rings), the frequency shift is twice the cavity bandwidth $(434 \mathrm{~Hz}$ for Qext $=3.10^{6}$ ). Thanks to a non-homogeneous copper coating ( $2 \mathrm{~mm}$ thick layer and $20 \mathrm{~mm}$ at the iris), a frequency shift @ $40 \mathrm{MV} / \mathrm{m}$ within the cavity bandwidth was obtained.

Table 3: Computed frequency shift for TESLA cavities at Eacc $=40 \mathrm{MV} / \mathrm{m}$ for different stiffening schemes.

\begin{tabular}{|c|c|}
\hline Configuration & $\Delta \mathbf{f}$ \\
\hline niobium $2.5 \mathrm{~mm}$ unstiffened & $-2135 \mathrm{~Hz}$ \\
\hline $\mathrm{Nb} 2.5 \mathrm{~mm}+$ EB welded stiffening rings & $\mathbf{- 8 6 3 ~ H z}$ \\
\hline $\mathrm{Nb} 2.5 \mathrm{~mm}+\mathrm{Cu}$ coating $2 \mathrm{~mm}$ & $-883 \mathrm{~Hz}$ \\
\hline $\begin{array}{c}\mathrm{Nb} 2.5 \mathrm{~mm}+\mathrm{Cu} \text { coating } 2 \mathrm{~mm}+\text { iris } \\
\text { stiffening }(\mathrm{h}=20 \mathrm{~mm})\end{array}$ & $\mathbf{- 3 5 8 ~ H z}$ \\
\hline
\end{tabular}

The Young modulus of the coating used with the $3 \mathrm{GHz}$ cavity prototypes was estimated to $27 \mathrm{GPa}$ from a simple experiment which consists in measuring the coated cavity deformation versus the applied axial force and comparison with a model calculation. Using this value, simulations on 9-cells TESLA cavities give a $\Delta \mathrm{f}$ of 616 $\mathrm{Hz}$ at $40 \mathrm{MV} / \mathrm{m}$ (non-homogeneous scheme), a value higher than the cavity bandwidth, but still an improvement as compared to the $863 \mathrm{~Hz}$ obtained with the actual EB welded stiffening rings. Simulations also show that this coating is efficient for accelerating fields up to $33 \mathrm{MV} / \mathrm{m}$. Different theoretical approaches modelled the effect of the porosity on the Young modulus. As the bulk copper Young modulus is $130 \mathrm{GPa}$, the estimated value $(27 \mathrm{GPa})$ is in the range of expected values of APS coating with porosity lying between $15 \%$ and $30 \%$. Mechanical model calculations show that to achieved an effective stiffening at $40 \mathrm{MV} / \mathrm{m}$, it is necessary to have a coating Young modulus of $95 \mathrm{GPa}$, which corresponds to a porosity of a few percent.

\section{CONCLUSION AND FUTURE}

A new fabrication method for SRF cavities is presented. The principle is to stiffen niobium cavities with a copper layer deposited by thermal spraying. Comparison of RF performances obtained with the first cavities tested before and after copper deposition showed that the maximum accelerating field was not affected, while a stiffening effect was measured. These results demonstrate the interest of the method. But this "rough APS" copper coating is efficent only for Eacc $<33 \mathrm{MV} / \mathrm{m}$. Mechanical simulations have proved that porosity have to be less than a few percent. Thermal measurements showed that for this coating at $40 \mathrm{MV} / \mathrm{m}$, the cavity thermal stability should not be affected if the bonding layer is removed.

This first study pointed out the important parameters involved in the cavity stiffening. Now, a new program has just started to investigate other spraying process (VPS, HVOF, and a more controlled APS), more suited to our application. After measurements of mechanical and thermal properties on samples, $3 \mathrm{GHz}$ and $1.3 \mathrm{GHz}$ cavities will be fabricated from $1 \mathrm{~mm}$ thick $\mathrm{Nb}$ sheets of RRR 130 and then stiffened with the copper layer.

\section{ACKNOWLEDGMENTS}

The authors would like to thank M.Boloré, A.Caruette, J.Y.Gasser, N.Hamoudi, A.Le Goff, J.C.Le Scornet, J.P.Poupeau, for their technical assistance and helpful discussions. They would like also to thank C.Coddet, C.Verdy (LERMPS) and V.Guipont, M.Jeandin (Ecole des Mines) for collaborating on plasma spraying.

\section{REFERENCES}

[1] W.D.Möller "The performance of the $1.3 \mathrm{GHz}$ superconducting RF cavities in the first module of the T.T.F linac" linac98, Chicago (1998).

[2] Lech Pawlowski " The Science and Engineering of Thermal Spray Coating " J.Wiley \& sons.

[3] M.Fouaidy et al. And reference here cited, " A New Fabrication and Stiffening Method of SRF Cavities " EPAC'98, Stockholm,(1998). 